\title{
Stochastic $\theta$-Methods for a Class of Jump-Diffusion Stochastic Pantograph Equations with Random Magnitude
}

\author{
Hua Yang ${ }^{1,2}$ and Feng Jiang ${ }^{3}$ \\ ${ }^{1}$ School of Automation, Huazhong University of Science and Technology, Wuhan 430074, China \\ ${ }^{2}$ School of Mathematics and Computer Science, Wuhan Polytechnic University, Wuhan 430023, China \\ ${ }^{3}$ School of Statistics and Mathematics, Zhongnan University of Economics and Law, Wuhan 430073, China \\ Correspondence should be addressed to Hua Yang; huay20@163.com and Feng Jiang; fjiang78@gmail.com
}

Received 30 August 2013; Accepted 21 October 2013; Published 6 February 2014

Academic Editors: N. Ganikhodjaev and Y. Sun

Copyright (C) 2014 H. Yang and F. Jiang. This is an open access article distributed under the Creative Commons Attribution License, which permits unrestricted use, distribution, and reproduction in any medium, provided the original work is properly cited.

\begin{abstract}
This paper is concerned with the convergence of stochastic $\theta$-methods for stochastic pantograph equations with Poisson-driven jumps of random magnitude. The strong order of the convergence of the numerical method is given, and the convergence of the numerical method is obtained. Some earlier results are generalized and improved.
\end{abstract}

\section{Introduction}

Recently, the study of stochastic pantograph equations (SPEs) has many results [1-3]. SPEs have been extensively applied in many fields such as finance, control, and engineering. However, in general, SPEs have no explicit solutions, and the study of numerical solutions of SPEs has received a great deal of attention. Fan et al. [4] investigate the $\alpha$ th moment asymptotical stability of the analytic solution and the numerical methods for the stochastic pantograph equation by using the Razumikhin technique. Baker and Buckwar [5] gave strong approximations to the solution obtained by a continuous extension of the $\theta$-Euler scheme and proved that the numerical solution produced by the continuous $\theta$-method converges to the true solution with order $1 / 2$. Fan et al. [6] investigated the existence and uniqueness of the solutions and convergence of semi-implicit Euler methods for stochastic pantograph equations under the local Lipschitz condition and the linear growth condition. Li et al. [7] investigated the convergence of the Euler method of the stochastic pantograph equations with Markovian switching under the weaker conditions. Reference [8] studied convergence and stability of numerical methods of stochastic pantograph differential equations.
In practice, stochastic differential equations with jump and numerical methods are also discussed extensively. In [9-13] strong convergence and mean-square stability properties were analysed in the case of Poisson-driven jumps of deterministic magnitude. References [14, 15] discussed the numerical methods of stochastic differential equations with random jump magnitudes. Motivated by the papers above, in this paper, we focus on stochastic pantograph equations with random jump magnitudes. SPEs with random jump magnitudes may be regarded as an extension of stochastic pantograph equations. Jump models arise in many other application areas and have proved successful at describing unexpected, abrupt changes of state [16-18]. Typically, these models do not admit analytical solutions and hence must be simulated numerically. Similar to stochastic differential equations [19-21], explicit solutions can hardly be obtained for SPEs with random jump magnitudes. Thus, appropriate numerical approximation schemes such as the Euler (or Euler-Maruyama) are needed to apply them in practice or to study their properties.

The paper is organised as follows. In Section 2, we introduce the SPEs with random jump magnitudes and define stochastic $\theta$-methods of (1). The main result of the paper is rather technical, so we present several lemmas in Section 3 and then complete the proof in Section 4. 


\section{Preliminaries}

Throughout this paper, we let $\left(\Omega, \mathscr{F},\left\{\mathscr{F}_{t}\right\}_{t \geq 0}, P\right)$ be a complete probability space with a filtration $\left\{\mathscr{F}_{t}\right\}_{t \geq 0}$ satisfying the usual conditions (i.e., it is increasing and right continuous while $\mathscr{F}_{0}$ contains all $P$-null sets $)$. Let $|\cdot|$ be the Euclidean norm in $\mathscr{R}^{n}$. Let $x_{0}$ be $\mathscr{F}_{0}$-measurable and right-continuous, and $E\left|x_{0}\right|^{2}<\infty$. Let $w(t)=\left(w_{t}^{1}, \ldots, w_{t}^{m}\right)^{T}$ be a $m$ dimensional Brownian motion defined on the probability space.

Consider a class of jump-diffusion stochastic pantograph equations with random magnitude of the form

$$
\begin{aligned}
d x(t)= & f\left(x\left(t^{-}\right), x\left(\mu t^{-}\right)\right) d t+g\left(x\left(t^{-}\right), x\left(\mu t^{-}\right)\right) d w(t) \\
& +h\left(x\left(t^{-}\right), x\left(\mu t^{-}\right), \gamma_{N\left(t^{-}\right)+1}\right) d N(t)
\end{aligned}
$$

on $0 \leq t \leq T$ with the initial value $x\left(0^{-}\right)=x_{0}$ and $0<$ $\mu<1$, where $N(t)$ is a Poisson process with mean $\lambda t ; x\left(t^{-}\right):=$ $\lim _{s \rightarrow t^{-}} x(s)$; and $\gamma_{i}, i=1,2, \ldots$ are independent, identically distributed random variables representing magnitudes for each jump.

Throughout, we assume that the jump magnitudes have bounded moments; that is, for some $q \geq 1$, there is a constant $B=B_{q}$ such that

$$
E\left(\left|\gamma_{i}\right|^{2 q}\right) \leq B
$$

We further employ the following assumptions.

Assumption 1. The functions $f, g$, and $h$ satisfy the global Lipschitz condition, that is, for each $k=1,2,3$, there is a positive constant $K_{1}$ such that

$$
\begin{aligned}
& \left|f\left(x_{1}, x_{2}\right)-f\left(y_{1}, y_{2}\right)\right|^{2} \vee\left|g\left(x_{1}, x_{2}\right)-f\left(y_{1}, y_{2}\right)\right|^{2} \\
& \quad \leq K_{1}\left(\left|x_{1}-y_{1}\right|^{2}+\left|x_{2}-y_{2}\right|^{2}\right) \\
& \left|h\left(x_{1}, x_{2}, x_{3}\right)-h\left(y_{1}, y_{2}, y_{3}\right)\right|^{2} \\
& \quad \leq K_{1}\left(\left|x_{1}-y_{1}\right|^{2}+\left|x_{2}-y_{2}\right|^{2}+\left|x_{3}-y_{3}\right|^{2}\right)
\end{aligned}
$$

where $x_{k}, y_{k} \in \mathfrak{R}^{n}$.

Assumption 2 (linear growth condition). There is a positive constant $K_{2}$ such that for all $t \in[0, T]$

$$
\begin{gathered}
\left|f\left(x_{1}, x_{2}\right)\right|^{2} \vee\left|g\left(x_{1}, x_{2}\right)\right|^{2} \leq K_{2}\left(1+\left|x_{1}\right|^{2}+\left|x_{2}\right|^{2}\right), \\
\left|h\left(x_{1}, x_{2}, x_{3}\right)\right|^{2} \leq K_{2}\left(1+\left|x_{1}\right|^{2}+\left|x_{2}\right|^{2}+\left|x_{3}\right|^{2}\right),
\end{gathered}
$$

for all $x_{1}, x_{2}, x_{3} \in \Re^{n}$.

In fact, the global Lipschitz condition (3) implies the linear growth condition (4). Under these conditions, it can be shown similarly as in [20] that (1) has a unique solution with all moments bounded.
We note for later reference that (1) involves the jump process

$$
\gamma(t):=\gamma_{N\left(t^{-}\right)+1}=\sum_{j=0}^{\infty} \gamma_{j+1} 1_{\left[\tau_{j}, \tau_{j+1}\right)}(t),
$$

where $\tau_{0}=0$ and $\tau_{j}, j=1,2, \ldots$ are the jump times.

One generalisation of stochastic $\theta$-Euler methods $[6,21]$ to system (1) has the form $y_{0}=x(0)$ and

$$
\begin{aligned}
y_{k+1}= & y_{k}+(1-\theta) f\left(y_{k}, y_{[\mu k]}\right) h+\theta f\left(y_{k+1}, y_{[\mu(k+1)]}\right) h \\
& +g\left(y_{k}, y_{[\mu k]}\right) \Delta w_{k}+h\left(y_{k}, y_{[\mu k]}, \gamma_{N\left(t_{k}\right)+1}\right) \Delta N_{k},
\end{aligned}
$$

where $\theta \in[0,1]$. Here $h \in(0,1)$ is a step size, which satisfies $M=T / h$ for some positive integer $M, t_{k}=k h(k=$ $0,1, \ldots, M) \cdot y_{k} \approx x\left(t_{k}\right), \Delta w_{k}=w\left(t_{k+1}\right)-w\left(t_{k}\right)$, and $\Delta N_{k}=$ $N\left(t_{k+1}\right)-N\left(t_{k}\right)$ are the Brownian and Poisson increments, respectively.

For $t \in\left[t_{k}, t_{k+1}\right]$, we define

$$
\begin{aligned}
y(t)= & y_{k}+(1-\theta) f\left(y_{k}, y_{[\mu k]}\right)\left(t-t_{k}\right) \\
& +\theta f\left(y_{k+1}, y_{[\mu(k+1)]}\right)\left(t-t_{k}\right) \\
& +g\left(y_{k}, y_{[\mu k]}\right)\left(w(t)-w\left(t_{k}\right)\right) \\
& +h\left(y_{k}, y_{[\mu k]}, \gamma_{N\left(t_{k}\right)+1}\right)\left(N(t)-N\left(t_{k}\right)\right)
\end{aligned}
$$

and denote

$$
\begin{gathered}
z_{1}(t)=\sum_{j=0}^{\infty} y_{j} 1_{[j h,(j+1) h)}(t), \\
z_{2}(t)=\sum_{j=0}^{\infty} y_{j+1} 1_{[j h,(j+1) h)}(t), \\
\bar{z}_{1}(t)=\sum_{j=0}^{\infty} y_{[\mu k]} 1_{[j h,(j+1) h)}(t), \\
\bar{z}_{2}(t)=\sum_{j=0}^{\infty} y_{[\mu(k+1)]} 1_{[j h,(j+1) h)}(t), \\
\bar{\gamma}(t)=\sum_{j=0}^{\infty} \gamma\left(t_{j}\right) 1_{[j h,(j+1) h)}(t) .
\end{gathered}
$$

Then, we define the continuous-time approximation

$$
\begin{aligned}
& y(t) \\
& =y_{0}+\int_{0}^{t}\left[(1-\theta) f\left(z_{1}(s), \bar{z}_{1}(s)\right)+\theta f\left(z_{2}(s), \bar{z}_{2}(s)\right)\right] d s \\
& \quad+\int_{0}^{t} g\left(z_{1}(s), \bar{z}_{1}(s)\right) d w(s) \\
& \quad+\int_{0}^{t} h\left(z_{1}(s), \bar{z}_{1}(s), \bar{\gamma}(s)\right) d N(s),
\end{aligned}
$$

which interpolates the discrete numerical approximation (6). So a convergence result for $y(t)$ immediately provides a result for $y_{k}$. 


\section{Lemmas}

Throughout our analysis, $C_{i}, D_{i}, i=1,2, \ldots$ denote generic constants, independent of $h$. The main theorem of the paper is rather technical. We will present a number of useful lemmas in the section and then complete the proof in Section 4.

Lemma 3. Under Assumption 2, there exists $0<h^{*}<1$ such that for all $0<h \leq h^{*}$,

$$
E\left|y_{k}\right|^{2} \leq C_{1}, \quad \text { for } k=1, \ldots, M .
$$

Proof. From (6), we have

$$
\begin{aligned}
E\left|y_{k+1}\right|^{2} \leq & 4 E\left|y_{k}\right|^{2} \\
& +4 E \mid(1-\theta) f\left(y_{k}, y_{[\mu k]}\right) h \\
& +\left.\theta f\left(y_{k+1}, y_{[\mu(k+1)]}\right) h\right|^{2} \\
& +4 E\left|g\left(y_{k}, y_{[\mu k]}\right) \Delta w_{k}\right|^{2} \\
& +4 E\left|h\left(y_{k}, y_{[\mu k]}, \gamma_{N\left(t_{k}\right)+1}\right) \Delta N_{k}\right|^{2} .
\end{aligned}
$$

Note that $2 \alpha \beta \leq|\alpha|^{2}+|\beta|^{2}$. Now, using Assumption 2,

$$
\begin{aligned}
E \mid & (1-\theta) f\left(y_{k}, y_{[\mu k]}\right) h+\left.\theta f\left(y_{k+1}, y_{[\mu(k+1)]}\right) h\right|^{2} \\
\leq & (1-\theta)^{2} h^{2} E\left|f\left(y_{k}, y_{[\mu k]}\right)\right|^{2}+\theta^{2} h^{2}\left|f\left(y_{k+1}, y_{[\mu(k+1)]}\right)\right|^{2} \\
& +(1-\theta) \theta h^{2} E\left[\left|f\left(y_{k}, y_{[\mu k]}\right)\right|^{2}+\left|f\left(y_{k+1}, y_{[\mu(k+1)]}\right)\right|^{2}\right] \\
\leq & K_{2}\left[(1-\theta)^{2} h^{2}+\theta^{2} h^{2}+(1-\theta) \theta h^{2}\right] \\
& +K_{2}\left[(1-\theta)^{2} h^{2}+(1-\theta) \theta h^{2}\right] E\left|y_{k}\right|^{2} \\
& +K_{2}\left[\theta^{2} h^{2}+(1-\theta) \theta h^{2}\right] E\left|y_{k+1}\right|^{2} \\
& +K_{2}\left[(1-\theta)^{2} h^{2}+(1-\theta) \theta h^{2}\right] E\left|y_{[\mu k]}\right|^{2} \\
& +K_{2}\left[\theta^{2} h^{2}+(1-\theta) \theta h^{2}\right] E\left|y_{[\mu(k+1)]}\right|^{2} .
\end{aligned}
$$

Using $E\left|\Delta w_{k}\right|^{2}=m h$ and Assumption 2, we have

$$
E\left|g\left(y_{k}, y_{[\mu k]}\right) \Delta w_{k}\right|^{2}=m h K_{2}\left(1+E\left|y_{k}\right|^{2}+E\left|y_{[\mu k]}\right|^{2}\right) \text {. }
$$

For the jump, we convert to the compensated Poisson increment $\Delta \widetilde{N}_{k}:=\Delta N_{k}-\lambda h$ with $E\left(\Delta \widetilde{N}_{k}\right)=0$ and $E\left(\Delta \widetilde{N}_{k}\right)^{2}=$ $\lambda h$ and Assumption 2. We then obtain

$$
\begin{aligned}
& E\left|h\left(y_{k}, y_{[\mu k]}, \gamma_{N\left(t_{k}\right)+1}\right) \Delta N(s)\right|^{2} \\
& \quad=E\left|h\left(y_{k}, y_{[\mu k]}, \gamma_{N\left(t_{k}\right)+1}\right)(\Delta \widetilde{N}(s)+\lambda h)\right|^{2} \\
& \quad=\lambda h(1+\lambda h) K_{2}\left(1+E\left|y_{k}\right|^{2}+E\left|y_{[\mu k]}\right|^{2}+E\left|\gamma_{N\left(t_{k}\right)+1}\right|^{2}\right) .
\end{aligned}
$$

Combining (13), (14), and (15) with (12) yields

$$
\begin{aligned}
E\left|y_{k+1}\right|^{2} \leq & A_{1}(h)+A_{2}(h) E\left|y_{k}\right|^{2}+A_{3}(h) E\left|y_{(k+1)}\right|^{2} \\
& +A_{4}(h) E\left|y_{[\mu k]}\right|^{2}+A_{5}(h) E\left|y_{[\mu(k+1)]}\right|^{2} \\
& +4 K_{2} \lambda h(1+\lambda h) E\left|\gamma_{N\left(t_{k}\right)+1}\right|^{2} \\
\leq & A_{1}(h)+A_{3}(h) E\left|y_{(k+1)}\right|^{2} \\
& +4 K_{2} \lambda h(1+\lambda h) E\left|\gamma_{N\left(t_{k}\right)+1}\right|^{2} \\
& +\left(A_{2}(h)+A_{4}(h)+A_{5}(h)\right) \max _{[\mu k] \leq i \leq k} E\left|y_{i}\right|^{2},
\end{aligned}
$$

where $A_{i}(h), i=1, \ldots, 5$ is a constant dependent on $h$ and $A_{3}(h)=4 K_{2} h^{2}\left(\theta^{2}+(1-\theta) \theta\right)$.

Now choosing $h$ sufficiently small such that $1-A_{3}(h) \geq$ $1 / 2$ and noting that (2) implies that each $E\left|\gamma\left(t_{j}\right)\right|^{2} \leq B_{1}$, we obtain

$$
\begin{aligned}
E\left|y_{k+1}\right|^{2} \leq & 2 A_{1}(h)+8 K_{2} \lambda h(1+\lambda h) B_{1} \\
& +2\left(A_{2}(h)+A_{4}(h)+A_{5}(h)\right) \max _{[\mu k] \leq i \leq k} E\left|y_{i}\right|^{2} .
\end{aligned}
$$

The result then follows from an application of the discrete Gronwall inequality. The proof is complete.

Lemma 4. Under Assumption 2, there exists $h^{*}>0$ such that, for all $0<h \leq h^{*}$,

$$
\begin{aligned}
& E\left|y(t)-z_{1}(t)\right|^{2} \leq C_{3} h, \quad \text { for } t \in[0, T], \\
& E\left|y(t)-z_{2}(t)\right|^{2} \leq C_{4} h, \quad \text { for } t \in[0, T] .
\end{aligned}
$$

Proof. Consider $t \in[k h,(k+1) h] \subseteq[0, T]$. In this interval we have

$$
\begin{aligned}
y(t)-z_{1}(t)= & y(t)-y_{k} \\
= & (1-\theta) f\left(y_{k}, y_{[\mu k]}\right)\left(t-t_{k}\right) \\
& +\theta f\left(y_{k+1}, y_{[\mu(k+1)]}\right)\left(t-t_{k}\right) \\
& +g\left(y_{k}, y_{[\mu k]}\right)\left(w(t)-w\left(t_{k}\right)\right) \\
& +h\left(y_{k}, y_{[\mu k]}, \gamma_{N\left(t_{k}\right)+1}\right)\left(N(t)-N\left(t_{k}\right)\right) .
\end{aligned}
$$

Thus,

$$
\begin{aligned}
E \mid y(t)- & \left.z_{1}(t)\right|^{2} \\
\leq & 3 E \mid(1-\theta) f\left(y_{k}, y_{[\mu k]}\right)\left(t-t_{n}\right) \\
& +\left.\theta f\left(y_{k+1}, y_{[\mu(k+1)]}\right)\left(t-t_{n}\right)\right|^{2} \\
& +3 E\left|g\left(y_{k}, y_{[\mu k]}\right)\left(w(t)-w\left(t_{k}\right)\right)\right|^{2} \\
& +3 E\left|h\left(y_{k}, y_{[\mu k]}, \gamma_{N\left(t_{k}\right)+1}\right)\left(N(t)-N\left(t_{k}\right)\right)\right|^{2} .
\end{aligned}
$$


Thus, by virtue of (12)-(14) and Lemma 3, we have

$$
\begin{aligned}
E\left|y(t)-z_{1}(t)\right|^{2} & \\
\leq & 3 m h^{2} K_{2}\left(1+2 C_{1}\right)+3 \lambda h(1+\lambda h) \\
& \times K_{2}\left(1+2 C_{1}+B_{1}\right)+15 K_{2} h^{2} \\
\leq & C_{3} h,
\end{aligned}
$$

where $C_{3}=3 m K_{2}\left(1+2 C_{1}\right)+3 \lambda(1+\lambda) K_{2}\left(1+2 C_{1}+B_{1}\right)+15 K_{2}$.

In a similar way we obtain (18). The proof is complete.

Lemma 5. Under Assumption 2, there exists $h^{*}>0$ such that, for all $0<h \leq h^{*}$,

$$
\begin{aligned}
& E\left|y(\mu t)-\bar{z}_{1}(t)\right|^{2} \leq C_{5} h, \quad \text { for } t \in[0, T], \\
& E\left|y(\mu t)-\bar{z}_{2}(t)\right|^{2} \leq C_{6} h \quad \text { for } t \in[0, T] .
\end{aligned}
$$

Proof. Consider $t \in[k h,(k+1) h] \subseteq[0, T]$. By (9), we have

$$
\begin{aligned}
y & (\mu t)-\bar{z}_{1}(t) \\
= & y(\mu t)-y_{[\mu k]} \\
= & y(\mu t)-y([\mu k] h) \\
= & \int_{[\mu k] h}^{\mu t}\left[(1-\theta) f\left(z_{1}(s), \bar{z}_{1}(s)\right)+\theta f\left(z_{2}(s), \bar{z}_{2}(s)\right)\right] d s \\
& +\int_{[\mu k] h}^{\mu t} g\left(z_{1}(s), \bar{z}_{1}(s)\right) d w(s) \\
& +\int_{[\mu k] h}^{\mu t} h\left(z_{1}(s), \bar{z}_{1}(s), \bar{\gamma}(s)\right) d N(s) .
\end{aligned}
$$

Thus,

$$
\begin{aligned}
& \left|y(\mu t)-\bar{z}_{1}(t)\right|^{2} \\
& \leq 3\left|\int_{[\mu k] h}^{\mu t}\left[(1-\theta) f\left(z_{1}(s), \bar{z}_{1}(s)\right)+\theta f\left(z_{2}(s), \bar{z}_{2}(s)\right)\right] d s\right|^{2} \\
& +3\left|\int_{[\mu k] h}^{\mu t} g\left(z_{1}(s), \bar{z}_{1}(s)\right) d w(s)\right|^{2} \\
& +3\left|\int_{[\mu k] h}^{\mu t} h\left(z_{1}(s), \bar{z}_{1}(s), \bar{\gamma}(s)\right) d N(s)\right|^{2} \\
& \leq 3\left|\int_{[\mu k] h}^{\mu t}\left[(1-\theta) f\left(z_{1}(s), \bar{z}_{1}(s)\right)+\theta f\left(z_{2}(s), \bar{z}_{2}(s)\right)\right] d s\right|^{2} \\
& +3\left|\int_{[\mu k] h}^{\mu t} g\left(z_{1}(s), \bar{z}_{1}(s)\right) d w(s)\right|^{2} \\
& +6\left|\int_{[\mu k] h}^{\mu t} h\left(z_{1}(s), \bar{z}_{1}(s), \bar{\gamma}(s)\right) d \widetilde{N}(s)\right|^{2} \\
& +6 \lambda^{2}\left|\int_{[\mu k] h}^{\mu t} h\left(z_{1}(s), \bar{z}_{1}(s), \bar{\gamma}(s)\right) d s\right|^{2} .
\end{aligned}
$$

Therefore, in view of the Hölder inequality and $\mu t-[\mu k] h \leq$ $2 h$, we have

$$
\begin{aligned}
& E\left|y(\mu t)-\bar{z}_{1}(t)\right|^{2} \\
& \leq 12 h E \int_{[\mu k] h}^{\mu t}\left[\left|f\left(z_{1}(s), \bar{z}_{1}(s)\right)\right|^{2}+\left|f\left(z_{2}(s), \bar{z}_{2}(s)\right)\right|^{2}\right] d s \\
& \quad+12 E\left|\int_{[\mu k] h}^{\mu t} g\left(z_{1}(s), \bar{z}_{1}(s)\right) d w(s)\right|^{2} \\
& \quad+24 E\left|\int_{[\mu k] h}^{\mu t} h\left(z_{1}(s), \bar{z}_{1}(s), \bar{\gamma}(s)\right) d \widetilde{N}(s)\right|^{2} \\
& \quad+12 h \lambda^{2} E \int_{[\mu k] h}^{\mu t}\left|h\left(z_{1}(s), \bar{z}_{1}(s), \bar{\gamma}(s)\right)\right|^{2} d s .
\end{aligned}
$$

Then, applying Itô and martingale isometries and Assumption 2, we have

$$
\begin{aligned}
& E\left|y(\mu t)-\bar{z}_{1}(t)\right|^{2} \\
& \leq 12 h K_{2} \int_{[\mu k] h}^{\mu t}\left[2+E\left|z_{1}(s)\right|^{2}+E\left|\bar{z}_{1}(s)\right|^{2}\right. \\
& \left.+E\left|z_{2}(s)\right|^{2}+E\left|\bar{z}_{2}(s)\right|^{2}\right] d s \\
& +12 K_{2} \int_{[\mu k] h}^{\mu t}\left(1+E\left|z_{1}(s)\right|^{2}+E\left|\bar{z}_{1}(s)\right|^{2}\right) d s \\
& +24 \lambda K_{2} \int_{[\mu k] h}^{\mu t}\left(1+E\left|z_{1}(s)\right|^{2}+E\left|\bar{z}_{1}(s)\right|^{2}+E|\bar{\gamma}(s)|^{2}\right) d s \\
& +12 h \lambda^{2} K_{2} \\
& \quad \times \int_{[\mu k] h}^{\mu t}\left(1+E\left|z_{1}(s)\right|^{2}+E\left|\bar{z}_{1}(s)\right|^{2}+E|\bar{\gamma}(s)|^{2}\right) d s .
\end{aligned}
$$

Now, note that (2) implies that each $E\left|\gamma\left(t_{j}\right)\right|^{2} \leq B_{1}$, on $[k h,(k+1) h], z_{1} \equiv y_{k}, z_{2} \equiv y_{k+1}, \bar{z}_{1} \equiv y_{k-m}, \bar{z}_{2} \equiv y_{k-m+1}$, and $\bar{\gamma} \equiv \gamma_{k}$. Hence, applying Lemma 3, we obtain

$$
\begin{aligned}
E\left|y(\mu t)-\bar{z}_{1}(t)\right|^{2} & \\
\leq & 48 K_{2} h^{2}\left(1+2 C_{1}\right)+24 K_{2} h\left(1+2 C_{1}\right) \\
& +48 K_{2} \lambda h\left(1+3 C_{1}\right)+24 K_{2} \lambda^{2} h^{2}\left(1+3 C_{1}\right) \\
\leq & C_{5} h,
\end{aligned}
$$


where $C_{5}=72 K_{2}\left(1+2 C_{1}\right)+24 K_{2} \lambda(2+\lambda)\left(1+3 C_{1}\right)$. In the following we consider $E\left|y(\mu t)-\bar{z}_{2}(t)\right|^{2}$ :

$$
\begin{aligned}
E \mid y(\mu t) & -\left.\bar{z}_{2}(t)\right|^{2} \\
& =E\left|y(\mu t)-y_{[\mu(k+1)]}\right|^{2} \\
& \leq 2 E\left|y(\mu t)-y_{[\mu k]}\right|^{2}+2 E\left|y_{[\mu k]}-y_{[\mu(k+1)]}\right|^{2} \\
& \leq 4 C_{5} h .
\end{aligned}
$$

Let $C_{6}=4 C_{5}$; the proof is complete.

\section{Main Results}

We can now state and prove our main result of this paper.

Theorem 6. Under Assumption 1 for some $q>1$ and Assumptions 1-2, there exists $h^{*}>0$ and $C=C(q)$ such that, for all $0<h<h^{*}$,

$$
E\left[\sup _{t \in[0, T]}|y(t)-x(t)|^{2}\right] \leq C h^{1-(1 / q)} .
$$

Proof. The analysis uses ideas from [15], where analogous results are derived in the stochastic differential equations. By construction, we have

$$
\begin{aligned}
& y(t)-x(t) \\
& =\int_{0}^{t}(1-\theta)\left[f\left(z_{1}(s), \bar{z}_{1}(s)\right)-f\left(x\left(s^{-}\right), x\left(\mu s^{-}\right)\right)\right] d s \\
& \quad+\int_{0}^{t} \theta\left[f\left(z_{2}(s), \bar{z}_{2}(s)\right)-f\left(x\left(s^{-}\right), x\left(\mu s^{-}\right)\right)\right] d s \\
& \quad+\int_{0}^{t}\left[g\left(z_{1}(s), \bar{z}_{1}(s)\right)-g\left(x\left(s^{-}\right), x\left(\mu s^{-}\right)\right)\right] d w(s) \\
& +\int_{0}^{t}\left[h\left(z_{1}(s), \bar{z}_{1}(s), \bar{\gamma}(s)\right)\right. \\
& \left.\quad-h\left(z_{1}(s), \bar{z}_{1}(s), \gamma\left(s^{-}\right)\right)\right] d N(s) \\
& +\int_{0}^{t}\left[h\left(z_{1}(s), \bar{z}_{1}(s), \gamma\left(s^{-}\right)\right)\right. \\
& \left.\quad-h\left(x\left(s^{-}\right), x\left(\mu s^{-}\right), \gamma\left(s^{-}\right)\right)\right] d N(s) .
\end{aligned}
$$

Now for any $0 \leq t_{1} \leq T$ we have

$$
\begin{array}{r}
E\left(\sup _{t \in\left[0, t_{1}\right]}|y(t)-x(t)|^{2}\right) \\
=4 E\left(\sup _{t \in\left[0, t_{1}\right]} \mid \int_{0}^{t}\left(( 1 - \theta ) \left[f\left(z_{1}(s), \bar{z}_{1}(s)\right)\right.\right.\right. \\
\left.-f\left(x\left(s^{-}\right), x\left(\mu s^{-}\right)\right)\right] \\
+\theta\left[f\left(z_{2}(s), \bar{z}_{2}(s)\right)\right. \\
\left.\left.\left.-f\left(x\left(s^{-}\right), x\left(\mu s^{-}\right)\right)\right]\right)\left.d s\right|^{2}\right)
\end{array}
$$

$$
\begin{gathered}
+4 E\left(\sup _{t \in\left[0, t_{1}\right]} \mid \int_{0}^{t}\left[g\left(z_{1}(s), \bar{z}_{1}(s)\right)\right.\right. \\
\left.\left.-g\left(x\left(s^{-}\right), x\left(\mu s^{-}\right)\right)\right]\left.d w(s)\right|^{2}\right) \\
+4 E\left(\sup _{t \in\left[0, t_{1}\right]} \mid \int_{0}^{t}\left[h\left(z_{1}(s), \bar{z}_{1}(s), \bar{\gamma}(s)\right)\right.\right. \\
+4 E\left(\sup _{t \in\left[0, t_{1}\right]} \mid \int_{0}^{t}\left[h\left(z_{1}(s), \bar{z}_{1}(s), \gamma\left(s^{-}\right)\right)\right.\right. \\
\left.\left.-h\left(z_{1}(s), \bar{z}_{1}(s), \gamma\left(s^{-}\right)\right)\right]\left.d N(s)\right|^{2}\right) \\
\left.\left.\left.-h\left(s^{-}\right), x\left(\mu s^{-}\right), \gamma\left(s^{-}\right)\right)\right]\left.d N(s)\right|^{2}\right) .
\end{gathered}
$$

By Assumption 1 and Hölder inequality, we have

$$
\begin{gathered}
E\left(\sup _{t \in\left[0, t_{1}\right]} \mid \int_{0}^{t}\left((1-\theta)\left[f\left(z_{1}(s), \bar{z}_{1}(s)\right)-f\left(x\left(s^{-}\right), x\left(\mu s^{-}\right)\right)\right]\right.\right. \\
+\theta\left[f\left(z_{2}(s), \bar{z}_{2}(s)\right)\right. \\
\left.\left.\left.-f\left(x\left(s^{-}\right), x\left(\mu s^{-}\right)\right)\right]\right)\left.d s\right|^{2}\right) \\
\leq 2 E\left(\operatorname { s u p } _ { t \in [ 0 , t _ { 1 } ] } \int _ { 0 } ^ { t } 1 ^ { 2 } d s \int _ { 0 } ^ { t _ { 1 } } \left(\mid f\left(z_{1}(s), \bar{z}_{1}(s)\right)\right.\right. \\
-\left.f\left(x\left(s^{-}\right), x\left(\mu s^{-}\right)\right)\right|^{2} \\
+\mid f\left(z_{2}(s), \bar{z}_{2}(s)\right) \\
\left.\left.-\left.f\left(x\left(s^{-}\right), x\left(\mu s^{-}\right)\right)\right|^{2}\right) d s\right) \\
\leq 2 T K_{1} \int_{0}^{t_{1}}\left(E\left|z_{1}(s)-x\left(s^{-}\right)\right|^{2}+E\left|\bar{z}_{1}(s)-x\left(\mu s^{-}\right)\right|^{2}\right. \\
\left.+E\left|z_{2}(s)-x\left(s^{-}\right)\right|^{2}+E\left|\bar{z}_{2}(s)-x\left(\mu s^{-}\right)\right|^{2}\right) d s .
\end{gathered}
$$

By Assumption 1, the Cauchy-Schwarz inequality, and the Doob inequality in the two martingale terms and the martingale isometry,

$$
\begin{aligned}
& E\left(\sup _{t \in\left[0, t_{1}\right]}\left|\int_{0}^{t}\left[g\left(z_{1}(s), \bar{z}_{1}(s)\right)-g\left(x\left(s^{-}\right), x\left(\mu s^{-}\right)\right)\right] d w(s)\right|^{2}\right) \\
& \quad \leq 4 E \int_{0}^{t_{1}}\left|g\left(z_{1}(s), \bar{z}_{1}(s)\right)-g\left(x\left(s^{-}\right), x\left(\mu s^{-}\right)\right)\right|^{2} d s \\
& \quad \leq 4 K_{1} \int_{0}^{t_{1}}\left[E\left|z_{1}(s)-x\left(s^{-}\right)\right|^{2}+E\left|\bar{z}_{1}(s)-x\left(\mu s^{-}\right)\right|^{2}\right] d s,
\end{aligned}
$$




$$
\begin{aligned}
& E\left(\sup _{t \in\left[0, t_{1}\right]} \mid \int_{0}^{t}\left[h\left(z_{1}(s), \bar{z}_{1}(s), \gamma\left(s^{-}\right)\right)\right.\right. \\
& \left.\left.-h\left(x\left(s^{-}\right), x\left(\mu s^{-}\right), \gamma\left(s^{-}\right)\right)\right]\left.d N(s)\right|^{2}\right) \\
& =E\left(\sup _{t \in\left[0, t_{1}\right]} \mid \int_{0}^{t}\left[h\left(z_{1}(s), \bar{z}_{1}(s), \gamma\left(s^{-}\right)\right)\right.\right. \\
& \left.-h\left(x\left(s^{-}\right), x\left(\mu s^{-}\right), \gamma\left(s^{-}\right)\right)\right] d \widetilde{N}(s) \\
& +\lambda \int_{0}^{t}\left[h\left(z_{1}(s), \bar{z}_{1}(s), \gamma\left(s^{-}\right)\right)\right. \\
& \left.\left.-h\left(x\left(s^{-}\right), x\left(\mu s^{-}\right), \gamma\left(s^{-}\right)\right)\right]\left.d s\right|^{2}\right) \\
& \leq 2 E\left(\sup _{t \in\left[0, t_{1}\right]} \mid \int_{0}^{t}\left[h\left(z_{1}(s), \bar{z}_{1}(s), \gamma\left(s^{-}\right)\right)\right.\right. \\
& \left.\left.-h\left(x\left(s^{-}\right), x\left(\mu s^{-}\right), \gamma\left(s^{-}\right)\right)\right]\left.d \widetilde{N}(s)\right|^{2}\right) \\
& +2 \lambda^{2} E\left(\sup _{t \in\left[0, t_{1}\right]} \mid \int_{0}^{t}\left[h\left(z_{1}(s), \bar{z}_{1}(s), \gamma\left(s^{-}\right)\right)\right.\right. \\
& \left.\left.-h\left(x\left(s^{-}\right), x\left(\mu s^{-}\right), \gamma\left(s^{-}\right)\right)\right]\left.d s\right|^{2}\right) \\
& \leq 8 E \mid \int_{0}^{t_{1}}\left[h\left(z_{1}(s), \bar{z}_{1}(s), \gamma\left(s^{-}\right)\right)\right. \\
& \left.-h\left(x\left(s^{-}\right), x\left(\mu s^{-}\right), \gamma\left(s^{-}\right)\right)\right]\left.d \widetilde{N}(s)\right|^{2} \\
& +2 \lambda^{2} T E\left(\int_{0}^{t_{1}} \mid h\left(z_{1}(s), \bar{z}_{1}(s), \gamma\left(s^{-}\right)\right)\right. \\
& \left.-\left.h\left(x\left(s^{-}\right), x\left(\mu s^{-}\right), \gamma\left(s^{-}\right)\right)\right|^{2} d s\right) \\
& \leq 8 \lambda E\left(\int_{0}^{t_{1}} \mid h\left(z_{1}(s), \bar{z}_{1}(s), \gamma\left(s^{-}\right)\right)\right. \\
& \left.-\left.h\left(x\left(s^{-}\right), x\left(\mu s^{-}\right), \gamma\left(s^{-}\right)\right)\right|^{2} d s\right) \\
& +2 \lambda^{2} T E\left(\int_{0}^{t_{1}} \mid h\left(z_{1}(s), \bar{z}_{1}(s), \gamma\left(s^{-}\right)\right)\right. \\
& \left.-\left.h\left(x\left(s^{-}\right), x\left(\mu s^{-}\right), \gamma\left(s^{-}\right)\right)\right|^{2} d s\right) \\
& \leq 8 \lambda K_{1} \int_{0}^{t_{1}}\left(E\left|z_{1}(s)-x\left(s^{-}\right)\right|^{2}+E\left|\bar{z}_{1}(s)-x\left(\mu s^{-}\right)\right|^{2}\right) d s \\
& +2 \lambda^{2} T K_{1} \int_{0}^{t_{1}}\left(E\left|z_{1}(s)-x\left(s^{-}\right)\right|^{2}+E\left|\bar{z}_{1}(s)-x\left(\mu s^{-}\right)\right|^{2}\right) d s . \\
& E\left(\sup _{t \in\left[0, t_{1}\right]} \mid \int_{0}^{t}\left[h\left(z_{1}(s), \bar{z}_{1}(s), \bar{\gamma}(s)\right)\right.\right. \\
& \left.\left.-h\left(z_{1}(s), \bar{z}_{1}(s), \gamma\left(s^{-}\right)\right)\right]\left.d N(s)\right|^{2}\right) \\
& =E\left(\sup _{t \in\left[0, t_{1}\right]} \mid \int_{0}^{t}\left[h\left(z_{1}(s), \bar{z}_{1}(s), \bar{\gamma}(s)\right)\right.\right. \\
& \left.-h\left(z_{1}(s), \bar{z}_{1}(s), \gamma\left(s^{-}\right)\right)\right] d \widetilde{N}(s) \\
& +\lambda \int_{0}^{t}\left[h\left(z_{1}(s), \bar{z}_{1}(s), \bar{\gamma}(s)\right)\right. \\
& \left.\left.-h\left(z_{1}(s), \bar{z}_{1}(s), \gamma\left(s^{-}\right)\right)\right]\left.d s\right|^{2}\right) \\
& \leq 2 E\left(\sup _{t \in\left[0, t_{1}\right]} \mid \int_{0}^{t}\left[h\left(z_{1}(s), \bar{z}_{1}(s), \bar{\gamma}(s)\right)\right.\right. \\
& \left.\left.-h\left(z_{1}(s), \bar{z}_{1}(s), \gamma\left(s^{-}\right)\right)\right]\left.d \widetilde{N}(s)\right|^{2}\right) \\
& +2 \lambda^{2} E\left(\sup _{t \in\left[0, t_{1}\right]} \mid \int_{0}^{t}\left[h\left(z_{1}(s), \bar{z}_{1}(s), \bar{\gamma}(s)\right)\right.\right. \\
& \left.\left.-h\left(z_{1}(s), \bar{z}_{1}(s), \gamma\left(s^{-}\right)\right)\right]\left.d s\right|^{2}\right) \\
& \leq 8 E \mid \int_{0}^{t_{1}}\left[h\left(z_{1}(s), \bar{z}_{1}(s), \bar{\gamma}(s)\right)\right. \\
& \left.-h\left(z_{1}(s), \bar{z}_{1}(s), \gamma\left(s^{-}\right)\right)\right]\left.d \widetilde{N}(s)\right|^{2} \\
& +2 \lambda^{2} T E\left(\int_{0}^{t_{1}} \mid h\left(z_{1}(s), \bar{z}_{1}(s), \bar{\gamma}(s)\right)\right. \\
& \left.-\left.h\left(z_{1}(s), \bar{z}_{1}(s), \gamma\left(s^{-}\right)\right)\right|^{2} d s\right) \\
& \leq 8 \lambda E\left(\int_{0}^{t_{1}} \mid h\left(z_{1}(s), \bar{z}_{1}(s), \bar{\gamma}(s)\right)\right. \\
& \left.-\left.h\left(z_{1}(s), \bar{z}_{1}(s), \gamma\left(s^{-}\right)\right)\right|^{2} d s\right) \\
& +2 \lambda^{2} T E\left(\int_{0}^{t_{1}} \mid h\left(z_{1}(s), \bar{z}_{1}(s), \bar{\gamma}(s)\right)\right. \\
& \left.-\left.h\left(z_{1}(s), \bar{z}_{1}(s), \gamma\left(s^{-}\right)\right)\right|^{2} d s\right)
\end{aligned}
$$




$$
\begin{aligned}
& \leq 2 \lambda(4+\lambda T) K_{1} E\left(\int_{0}^{t_{1}}\left|\bar{\gamma}(s)-\gamma\left(s^{-}\right)\right|^{2} d s\right) \\
& \leq 2 \lambda(4+\lambda T) K_{1}\left(\sum_{n=0}^{M^{\prime}-1} E\left(\int_{t_{n}}^{t_{n+1}}\left|\bar{\gamma}(s)-\gamma\left(s^{-}\right)\right|^{2} d s\right)\right),
\end{aligned}
$$

where $M^{\prime}$ is the smallest integer such that $M^{\prime} h \geq t_{1}$.

Now the number of nonzero terms in the summation in (34) is a random variable that is not independent of the summands. To obtain a useful bound, we recall the following Young's inequality:

$$
a b \leq \frac{q-1}{q} \varepsilon^{1 /(q-1)} a^{q /(q-1)}+\frac{1}{q \varepsilon} b^{q},
$$

where $a, b, \varepsilon>0$ and $1<q<\infty$.

Hence,

$$
\begin{aligned}
E\left(\int_{t_{n}}^{t_{n+1}}\left|\bar{\gamma}(s)-\gamma\left(s^{-}\right)\right|^{2} d s\right) \\
=E\left(1_{\left\{\Delta N_{n} \geq 1\right\}} \int_{t_{n}}^{t_{n+1}}\left|\bar{\gamma}(s)-\gamma\left(s^{-}\right)\right|^{2} d s\right) \\
\leq \frac{q-1}{q} \varepsilon^{1 /(q-1)} E\left(1_{\left\{\Delta N_{n} \geq 1\right\}}\right) \\
\quad+\frac{1}{q \varepsilon} E\left(\int_{t_{n}}^{t_{n+1}}\left|\bar{\gamma}(s)-\gamma\left(s^{-}\right)\right|^{2} d s\right)^{q} .
\end{aligned}
$$

Now we can apply the Hölder inequality as follows:

$$
\begin{aligned}
& E\left(\int_{t_{n}}^{t_{n+1}}\left|\bar{\gamma}(s)-\gamma\left(s^{-}\right)\right|^{2} d s\right)^{q} \\
& \quad \leq E\left[\left(\int_{t_{n}}^{t_{n+1}} d s\right)^{q-1} \int_{t_{n}}^{t_{n+1}}\left|\bar{\gamma}(s)-\gamma\left(s^{-}\right)\right|^{2 q} d s\right] \\
& \quad=h^{q-1} E\left[\int_{t_{n}}^{t_{n+1}}\left|\bar{\gamma}(s)-\gamma\left(s^{-}\right)\right|^{2 q} d s\right] .
\end{aligned}
$$

Using (37) in (35), we have

$$
\begin{aligned}
& E\left[\left|\bar{\gamma}(s)-\gamma\left(s^{-}\right)\right|^{2 q} d s\right] \\
& \quad \leq 2^{2 q-1}\left(E\left[|\bar{\gamma}(s)|^{2 q}\right]+E\left[\left|\gamma\left(s^{-}\right)\right|^{2 q}\right]\right),
\end{aligned}
$$

which follows from the Hölder inequality and (2); we yield

$$
\begin{aligned}
E\left(\int_{t_{n}}^{t_{n+1}}\left|\bar{\gamma}(s)-\gamma\left(s^{-}\right)\right|^{2} d s\right) \\
\leq \frac{q-1}{q} \varepsilon^{1 /(q-1)} E\left(1_{\left\{\Delta N_{n} \geq 1\right\}}\right) \\
\quad+\frac{h^{q-1}}{q \varepsilon} E\left(\int_{t_{n}}^{t_{n+1}}\left|\bar{\gamma}(s)-\gamma\left(s^{-}\right)\right|^{2 q} d s\right)
\end{aligned}
$$

$$
\begin{aligned}
\leq & \frac{q-1}{q} \varepsilon^{1 /(q-1)} P\left(\Delta N_{n} \geq 1\right) \\
& +\frac{h^{q-1}}{q \varepsilon} \int_{t_{n}}^{t_{n+1}} E\left|\bar{\gamma}(s)-\gamma\left(s^{-}\right)\right|^{2 q} d s \\
\leq & \frac{q-1}{q} \varepsilon^{1 /(q-1)} \lambda h \\
& +\frac{h^{q-1}}{q \varepsilon} 2^{2 q-1} \int_{t_{n}}^{t_{n+1}}\left(E|\bar{\gamma}(s)|^{2 q}+E\left|\gamma\left(s^{-}\right)\right|^{2 q}\right) d s \\
\leq & \frac{q-1}{q} \varepsilon^{1 /(q-1)} \lambda h+\frac{h^{q} B}{q \varepsilon} 2^{2 q} .
\end{aligned}
$$

Choosing $\varepsilon=h^{(q-1)^{2} / q}$, we have

$$
E\left(\int_{t_{n}}^{t_{n+1}}\left|\bar{\gamma}(s)-\gamma\left(s^{-}\right)\right|^{2} d s\right) \leq \frac{1}{q}\left((q-1) \lambda+2^{2 q} B\right) h^{2-1 / q} .
$$

Substituting (40) into (34) yields

$$
\begin{aligned}
& E\left(\sup _{t \in\left[0, t_{1}\right]} \mid \int_{0}^{t}\left[h\left(z_{1}(s), \bar{z}_{1}(s), \bar{\gamma}(s)\right)\right.\right. \\
& \left.\left.\quad-h\left(z_{1}(s), \bar{z}_{1}(s), \gamma\left(s^{-}\right)\right)\right]\left.d N(s)\right|^{2}\right) \\
& \leq 2 \lambda(4+\lambda T) K_{1} \sum_{n=0}^{M-1} \frac{1}{q}\left((q-1) \lambda+2^{2 q} B\right) h^{2-1 / q} \\
& \leq \frac{2 \lambda}{q}(4+\lambda T) K_{1}\left((q-1) \lambda+2^{2 q} B\right) M h^{2-1 / q} \\
& \leq \frac{2 \lambda}{q}(4+\lambda T) T K_{1}\left((q-1) \lambda+2^{2 q} B\right) h^{1-1 / q}
\end{aligned}
$$

as $M h \leq T$.

Now, substituting (31), (32), (33), and (41) into (30) yields

$$
\begin{aligned}
& E\left(\sup _{t \in\left[0, t_{1}\right]}|y(t)-x(t)|^{2}\right) \\
& \leq \frac{8 \lambda T K_{1}}{q}(4+\lambda T)\left((q-1) \lambda+2^{2 q} B\right) h^{1-1 / q} \\
& \quad+8 K_{1}\left(T+2+4 \lambda+\lambda^{2} T\right) \int_{0}^{t_{1}} E\left|z_{1}(s)-x\left(s^{-}\right)\right|^{2} d s \\
& \quad+8 K_{1}\left(T+2+4 \lambda+\lambda^{2} T\right) \int_{0}^{t_{1}} E\left|\bar{z}_{1}(s)-x\left(\mu s^{-}\right)\right|^{2} d s \\
& \quad+8 T K_{1} \int_{0}^{t_{1}} E\left|z_{2}(s)-x\left(s^{-}\right)\right|^{2} d s \\
& \quad+8 T K_{1} \int_{0}^{t_{1}} E\left|\bar{z}_{2}(s)-x\left(\mu s^{-}\right)\right|^{2} d s
\end{aligned}
$$




$$
\begin{aligned}
\leq & \frac{8 \lambda T K_{1}}{q}(4+\lambda T)\left((q-1) \lambda+2^{2 q} B\right) h^{1-1 / q} \\
& +16 K_{1}\left(T+2+4 \lambda+\lambda^{2} T\right) \\
& \times \int_{0}^{t_{1}}\left(E\left|z_{1}(s)-y(s)\right|^{2}+E\left|y(s)-x\left(s^{-}\right)\right|^{2}\right) d s \\
& +16 K_{1}\left(T+2+4 \lambda+\lambda^{2} T\right) \\
& \times \int_{0}^{t_{1}}\left(E\left|\bar{z}_{1}(s)-y(s-\tau)\right|^{2}+E\left|y(s-\tau)-x\left(\mu s^{-}\right)\right|^{2}\right) d s \\
& +16 T K_{1} \int_{0}^{t_{1}}\left(E\left|z_{2}(s)-y(s)\right|^{2}+E\left|y(s)-x\left(s^{-}\right)\right|^{2}\right) d s \\
& +16 T K_{1} \int_{0}^{t_{1}}\left(E\left|\bar{z}_{2}(s)-y(s-\tau)\right|^{2}\right. \\
& \left.+E\left|y(s-\tau)-x\left(\mu s^{-}\right)\right|^{2}\right) d s .
\end{aligned}
$$

From Lemmas 4 and 5, we have

$$
\begin{aligned}
& E\left(\sup _{t \in\left[0, t_{1}\right]}|y(t)-x(t)|^{2}\right) \\
& \leq \frac{8 \lambda T K_{1}}{q}(4+\lambda T)\left((q-1) \lambda+2^{2 q} B\right) h^{1-1 / q} \\
& \quad+16 K_{1} C_{3}\left(T+2+4 \lambda+\lambda^{2} T\right) h \\
& \quad+16 K_{1} C_{5}\left(T+2+4 \lambda+\lambda^{2} T\right) h \\
& \quad+16 T K_{1} C_{4} h+16 T K_{1} C_{6} h+32 K_{1}\left(2 T+2+4 \lambda+\lambda^{2} T\right) \\
& \quad \times \int_{0}^{t_{1}} E \sup _{t \in[0, s]}\left|y(t)-x\left(t^{-}\right)\right|^{2} d s \\
& \leq 32 K_{1}\left(2 T+2+4 \lambda+\lambda^{2} T\right) \\
& \quad \times \int_{0}^{t_{1}} E \sup _{t \in[0, s]}\left|y(t)-x\left(t^{-}\right)\right|^{2} d s+D_{1} h^{1-1 / q},
\end{aligned}
$$

where $D_{1}:=\left(8 \lambda T K_{1} / q\right)(4+\lambda T)\left((q-1) \lambda+2^{2 q} B\right)+16 K_{1}\left(\left(C_{3}+\right.\right.$ $\left.\left.C_{5}\right)\left(T+2+4 \lambda+\lambda^{2} T\right)+T C_{4}+T C_{6}\right)$.

By the Gronwall inequality, we have

$$
E\left(\sup _{t \in[0, T]}|y(t)-x(t)|^{2}\right) \leq D_{1} h^{1-1 / q} e^{32 K_{1}\left(2 T+2+4 \lambda+\lambda^{2} T\right)}
$$

The proof is complete.

Remark 7. Theorem 6 shows that the order of convergence in mean square is close to 1 . Moreover, stochastic $\theta$-methods give strong convergence rate arbitrarily close to order $1 / 2$ under appropriate moment bounds on the jump magnitude.
This problem class is now widely used in mathematical finance.

By Theorem 6, we obtain the following corollaries.

Corollary 8. Under Assumption 1,

$$
\lim _{h \rightarrow 0} E\left[\sup _{t \in[0, T]}|y(t)-x(t)|^{2}\right]=0 \text {. }
$$

The convergent result can be extended to the case of nonlinear coefficients that are local Lipschitz $[6,7,12]$ based on the style of analysis in [22].

Corollary 9. Under the local Lipschitz condition and Assumption 2,

$$
\lim _{h \rightarrow 0} E\left[\sup _{t \in[0, T]}|y(t)-x(t)|^{2}\right]=0 .
$$

Remark 10. Corollary 9 shows that the numerical solution converges to the true solution. However, the order of the convergence of the numerical method is not given under the local Lipschitz condition. If we remove jump and discuss the system without time lag, our results are reduced to the results derived in $[6,14]$. In other words, our results are the generalization of paper $[6,14]$.

\section{Conflict of Interests}

The authors declare that there is no conflict of interests regarding the publication of this paper.

\section{Acknowledgments}

The work is supported by the Fundamental Research Funds for the Central Universities, the National Natural Science Foundation of China under Grant 61304067, 11271146 and 61304175 the Natural Science Foundation of Hubei Province of China under Grant 2013CFB443.

\section{References}

[1] X. Meng, S. Hu, and P. Wu, "Pathwise estimation of stochastic differential equations with unbounded delay and its application to stochastic pantograph equations," Acta Applicandae Mathematicae, vol. 113, no. 2, pp. 231-246, 2011.

[2] Z. Yan and Y. Huang, "Robust $H_{\infty}$ lter design for Itô stochastic pantograph systems," Mathematical Problems in Engineering, vol. 2013, Article ID 747890, 8 pages, 2013.

[3] J. A. D. Appleby and E. Buckwar, "Sufficient condition for polynomial asymptotic behavior of the stochastic pantograph equation," http://www4.dcu.ie/maths/research/preprint.shtml.

[4] Z. Fan, M. Song, and M. Liu, "The $\alpha$ th moment stability for the stochastic pantograph equation," Journal of Computational and Applied Mathematics, vol. 233, no. 2, pp. 109-120, 2009.

[5] C. T. H. Baker and E. Buckwar, "Continuous $\Theta$-methods for the stochastic pantograph equation," Electronic Transactions on Numerical Analysis, vol. 11, pp. 131-151, 2000.

[6] Z. Fan, M. Liu, and W. Cao, "Existence and uniqueness of the solutions and convergence of semi-implicit Euler methods 
for stochastic pantograph equations," Journal of Mathematical Analysis and Applications, vol. 325, no. 2, pp. 1142-1159, 2007.

[7] R. Li, M. Liu, and W. K. Pang, "Convergence of numerical solutions to stochastic pantograph equations with Markovian switching," Applied Mathematics and Computation, vol. 215, no. 1, pp. 414-422, 2009.

[8] Y. Xiao and H. Zhang, "Convergence and stability of numerical methods with variable step size for stochastic pantograph differential equations," International Journal of Computer Mathematics, vol. 88, no. 14, pp. 2955-2968, 2011.

[9] D. J. Higham and P. E. Kloeden, "Numerical methods for nonlinear stochastic differential equations with jumps," Numerische Mathematik, vol. 101, no. 1, pp. 101-119, 2005.

[10] D. J. Higham and P. E. Kloeden, "Strong convergence rates for backward Euler on a class of nonlinear jump-diffusion problems," Journal of Computational and Applied Mathematics, vol. 205, no. 2, pp. 949-956, 2007.

[11] D. J. Higham and P. E. Kloeden, "Convergence and stability of implicit methods for jump-diffusion systems," International Journal of Numerical Analysis \& Modeling, vol. 3, pp. 125-140, 2006.

[12] L. S. Wang, C. Mei, and H. Xue, "The semi-implicit Euler method for stochastic differential delay equation with jumps," Applied Mathematics and Computation, vol. 192, no. 2, pp. 567578, 2007.

[13] L. Ronghua, M. Hongbing, and D. Yonghong, "Convergence of numerical solutions to stochastic delay differential equations with jumps," Applied Mathematics and Computation, vol. 172, no. 1, pp. 584-602, 2006.

[14] G. D. Chalmers and D. J. Higham, "Convergence and stability analysis for implicit simulations of stochastic differential equations with random jump magnitudes," Discrete and Continuous Dynamical Systems B, vol. 9, no. 1, pp. 47-64, 2008.

[15] F. Jiang, Y. Shen, and L. Liu, "Numerical methods for a class of jump-diffusion systems with random magnitudes," Communications in Nonlinear Science and Numerical Simulation, vol. 16, no. 7, pp. 2720-2729, 2011.

[16] S. G. Kou, "A jump-diffusion model for option pricing," Management Science, vol. 48, no. 8, pp. 1086-1101, 2002.

[17] A. Svishchuk and A. Kalemanova, "The stochastic stability of interest rates with jump changes," Theory of Probability and Mathematical Statistics, vol. 61, pp. 161-172, 2000.

[18] R. Cont and P. Tankov, Financial Modelling with Jump Processes, Chapman \& Hall/CRC, Boca Raton, Fa, USA, 2004.

[19] F. Jiang, Y. Shen, and F. Wu, "A note on order of convergence of numerical method for neutral stochastic functional differential equations," Communications in Nonlinear Science and Numerical Simulation, vol. 17, no. 3, pp. 1194-1200, 2012.

[20] A. Gardon', "The order of approximations for solutions of Itôtype stochastic differential equations with jumps," Stochastic Analysis and Applications, vol. 22, no. 3, pp. 679-699, 2004.

[21] P. Hu and C. Huang, "Stability of stochastic $\theta$-methods for stochastic delay integro-differential equations," International Journal of Computer Mathematics, vol. 88, no. 7, pp. 1417-1429, 2011.

[22] X. Mao and S. Sabanis, "Numerical solutions of stochastic differential delay equations under local Lipschitz condition," Journal of Computational and Applied Mathematics, vol. 151, no. 1, pp. 215-227, 2003. 


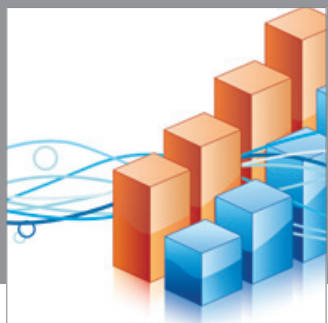

Advances in

Operations Research

mansans

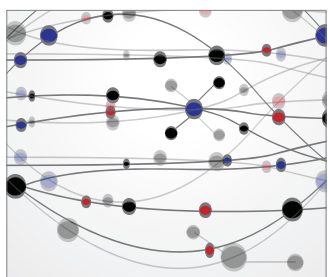

The Scientific World Journal
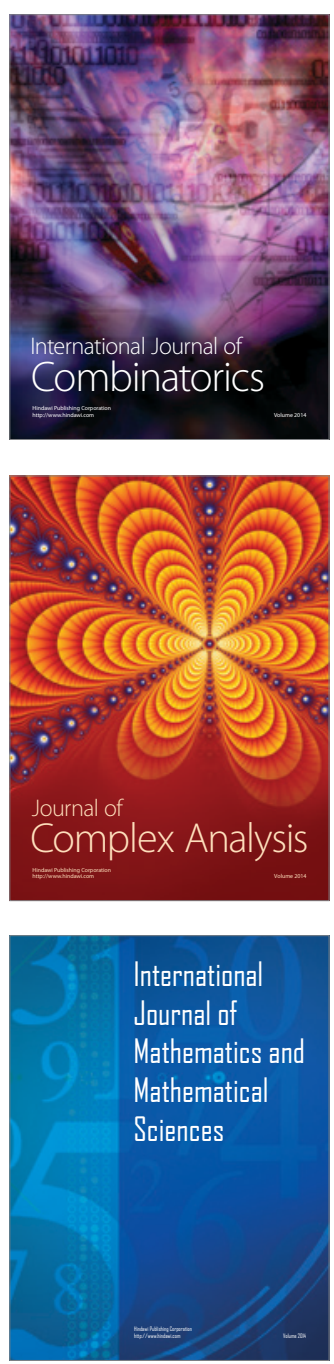
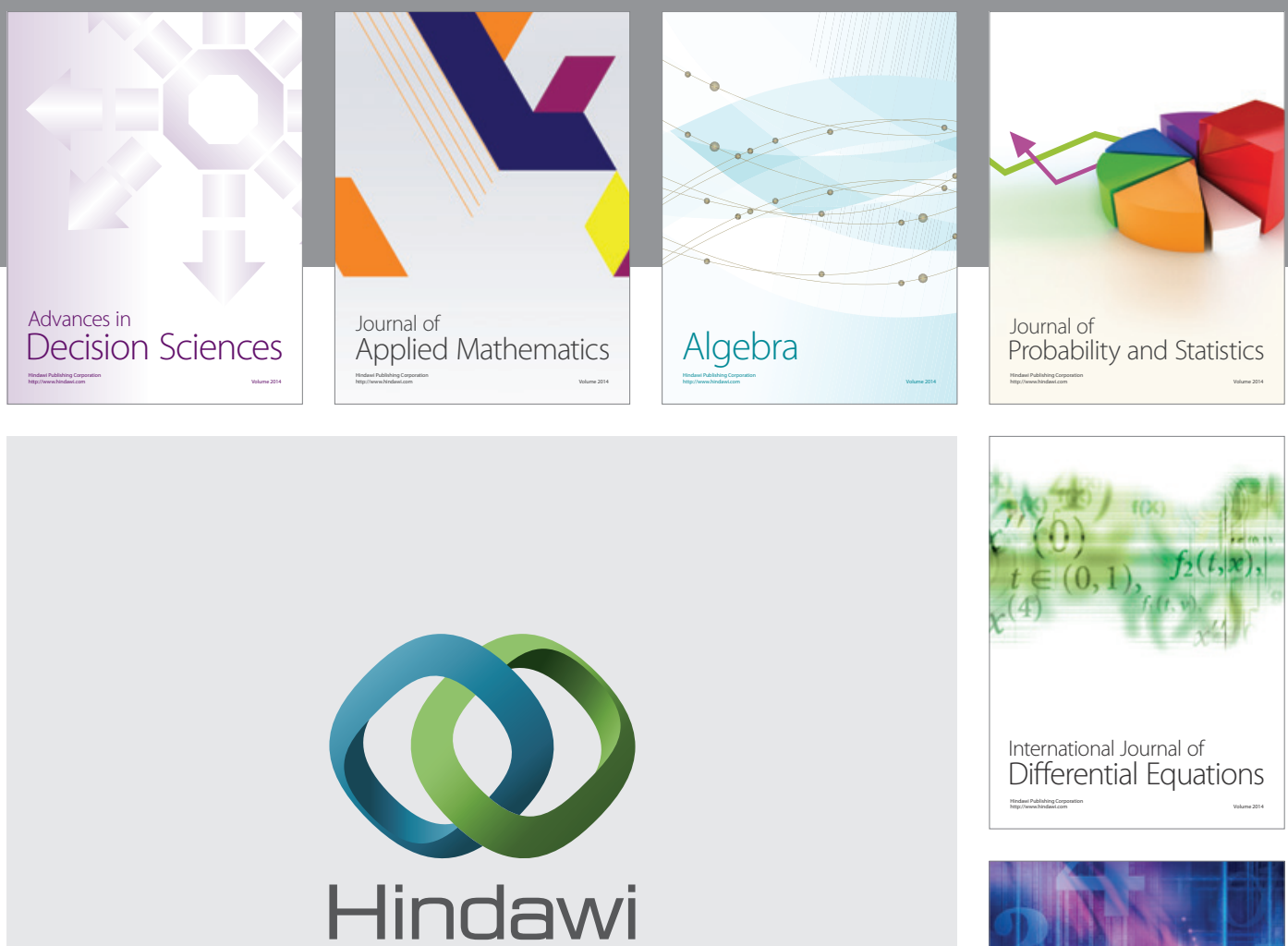

Submit your manuscripts at http://www.hindawi.com
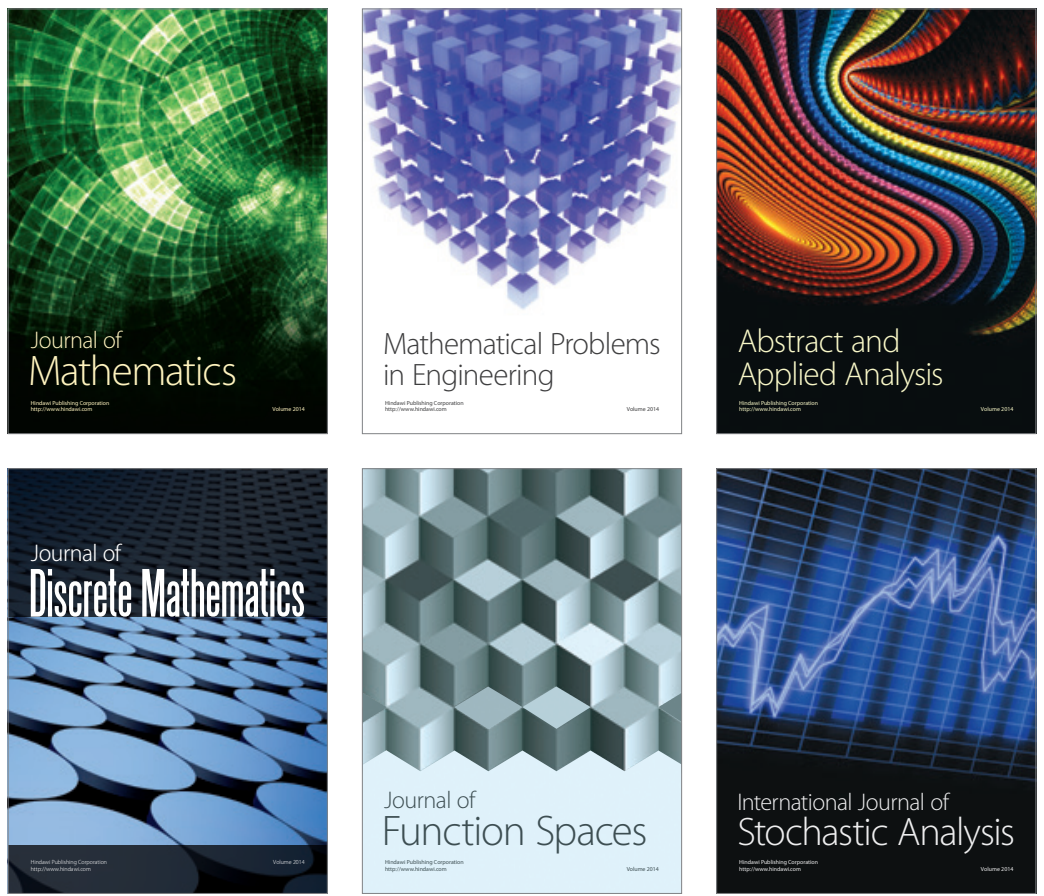

Journal of

Function Spaces

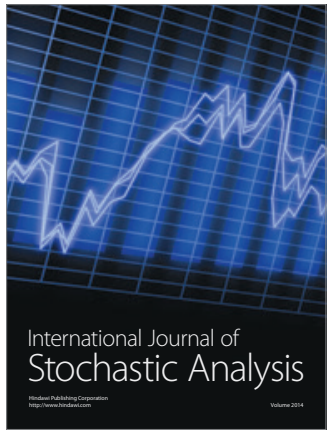

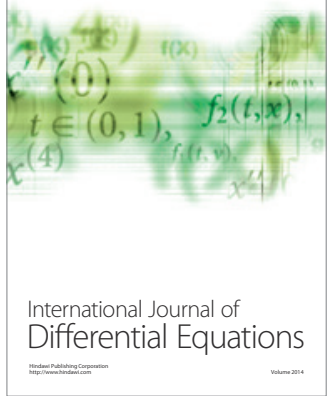
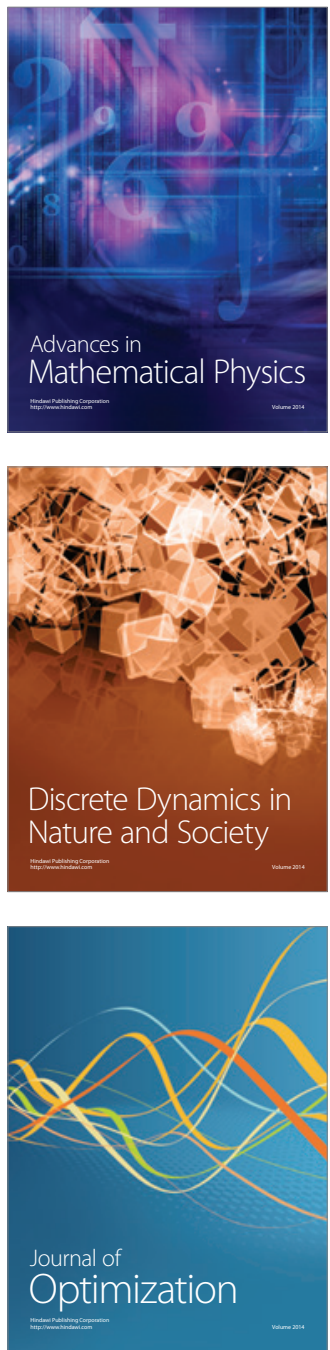